\title{
Effect of Reaction Rate on Dispersion of Atmospheric Aerosols in the Presence of Electric Field
}

\author{
Meena Priya.P \\ Mathematics Section, Faculty of \\ Engineering and Technology, \\ Annamalai University, India
}

\author{
Nirmala P. Ratchagar \\ Mathematics Section, Faculty of \\ Engineering and Technology, \\ Annamalai University, India
}

\begin{abstract}
Under the effects of electric field and chemical reaction, a mathematical model has been developed to study the dispersion of aerosols in a couple stress fluid flowing through a channel which is analytically solved using Taylor dispersion model. The solutions for velocity, concentration distribution and dispersion coefficient are numerically computed and the results are represented graphically. It reveals that the dispersion coefficient increases with increase in electric number but decreases with increase in couple stress parameter and chemical reaction rate.
\end{abstract}

Keywords

electrohydrodynamics, pollution, couple stress fluid

\section{INTRODUCTION}

Emission of air pollutants is caused by different anthropogenic processes which can be categorized into the source groups motor traffic, industry, power-plants, trade and domestic fuel [1]. All liquid or solid particles suspended in air are defined as aerosol particles. These particles influence climate, cloud formation, weather, visibility, atmospheric chemistry as well as human health. Two groups of aerosol particles are distinguished: primary particles are those that have direct sources. Secondary particles are produced in the atmosphere by gas-to-particle conversion. Sometimes, the primary pollutants may not cause pollution of the air but secondary pollutants produce air borne pollutants [5]. In recent years, the study of a chemically reactive heavy admixture and its by-product has generated considerable attention because of its several harmful effects on human society and its environment.

In the nature transition between ionosphere and atmosphere of the earth is a region having poorly conducting fluid in which the electrical forces dominate in driving the fluid [4]. Further the electrical forces in thunderstorm also appear to be as important as the fluid forces at some stages of critical forces [2]. Besides the various electrical phenomena, it is found that different chemical compositions of aerosols take place nearearth's atmosphere [3]. Fluids with antisymmetric stress are known as polar fluids. Couple stress fluids are specialized micropolar fluid where in unlike most micropolar fluids, there is a match between the spin of the suspension and the vorticity of the suspending fluid [9]. The aerosol particles can collide with other particles and grow into large enough to be removed by gravity or electrical [6]. The process by which these particles migrate, either to a surface or to one another, is called diffusion and their motion is described as dispersion [7]. The dispersion of aerosols in the atmosphere by gravity or electrical have significant impact on the human system [8]. So, it is imperative to have a mathematical model to consider secondary pollutant due to chemical reaction and their removal by means of electrical. This paper deals with the effect of electric field and chemical reaction on the dispersion of aerosols in a couple stress fluid flowing in a channel using Taylor's model.

\section{MATHEMATICAL FORMULATION}

The physical configuration shown in the figure 1 consists of an infinite horizontal channel bounded on both sides by electro-conducting impermeable rigid plates embeded with electrodes located at $\mathrm{y}=0$ and $\mathrm{y}=\mathrm{h}$ and electric potentials $\phi=\frac{V}{h} x$ at $\mathrm{y}=0$ and $\phi=\frac{V}{h}\left(x-x_{0}\right)$ at $\mathrm{y}=\mathrm{h}$ are maintained on these boundaries where $\mathrm{V}$ is potential.

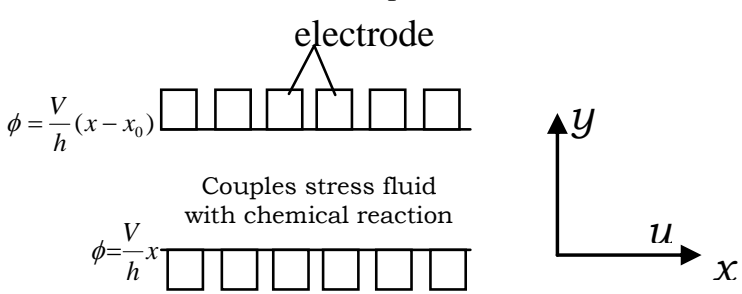

Figure 1. Physical Configuration

In this paper, we make the assumption that the electrical conductivity $(\sigma)$ is negligibly small and hence the magnetic field is negligible. This assumption makes the electric field $\overrightarrow{\mathrm{E}}$, to be conservative.

$$
\text { i.e. } \vec{E}=-\nabla \phi
$$

The basic equations are,

Conservation of mass for an incompressible flow

$$
\nabla \cdot \overrightarrow{\mathrm{q}}=0
$$

Conservation of momentum

$$
\begin{aligned}
& \rho\left(\frac{\partial \vec{q}}{\partial t}+(\vec{q} \cdot \nabla) \vec{q}\right)=-\nabla p+\mu \nabla^{2} \vec{q}-\lambda \nabla^{4} \vec{q} \\
& +\rho_{e} \vec{E}
\end{aligned}
$$


where $\lambda$ is a couple stress parameter.

Conservation of species

$$
\frac{\partial C}{\partial t}+(\vec{q} \cdot \nabla) C=D \nabla^{2} C
$$

\section{Conservation of charges}

$\frac{\partial \rho_{e}}{\partial t}+(\vec{q} . \nabla) \rho_{e}+\nabla . \vec{J}=0$

Maxwell's equation

$\nabla . \vec{E}=\frac{\rho_{e}}{\varepsilon_{0}}($ Gauss law $)$

(6)

$\nabla \times \vec{E}=0$ (Faraday 's law)

The equation (7) is zero because in a poorly conducting fluid, induced magnetic field is negligible and there is no applied magnetic field.

$\vec{J}=\sigma \vec{E}\left(\mathrm{Ohm} s^{\prime}\right.$ law $)$

The above equations are solved using the following boundary conditions on velocity and potential,

$u=0 \quad$ at $\quad y=0, h$

couple stress condition, $\quad \frac{d^{2} u}{d y^{2}}=0^{\text {at }} \mathrm{y}=0, \mathrm{~h}$

$\phi=\frac{V}{h} x$ at $y=0$

$\phi=\frac{V}{h}\left(x-x_{0}\right)$ at $\left.y=h\right\}$

In cartesian form, using the above approximation equation (3) becomes

$0=-\frac{\partial p}{\partial x}+\mu \nabla^{2}-\lambda \nabla^{4} u+\rho_{e} E_{x}, \nabla^{2}=\frac{\partial^{2}}{\partial y^{2}}$

In a poorly conducting fluid, the electrical conductivity is assumed to vary linearly with temperature in the form

$\sigma=\sigma_{0}\left[1+\alpha_{h}\left(T_{b}-T_{0}\right)\right]$

where $\alpha_{h}$ is the coefficient of volumetric expansion.

We assume the flow is fully developed and unidirectional in the $\mathrm{x}$-direction, so that the velocity and temperature will be functions of $\mathrm{y}$ only. Using the following dimensionless quantities,

$$
y^{*}=\frac{y}{h} ; u^{*}=\frac{u}{\frac{v}{h}} ; E_{x} *=\frac{E_{x}}{\frac{V}{h}} ; \rho_{e}{ }^{*}=\frac{\rho_{e}}{\frac{\varepsilon_{0} V}{h^{2}}} ; P^{*}=\frac{P}{\rho\left(\frac{v}{h}\right)^{2}} ; x^{*}=\frac{x}{h}
$$

where $\mathrm{V}$ is electric potential. We get electric potential through electrodes.

Equations (3) to (11) becomes

$\frac{d^{4} u}{d y^{4}}-\frac{\mu}{\lambda} h^{2} \frac{d^{2} u}{d y^{2}}-\frac{\rho_{e} E_{x} \varepsilon_{0} V^{2} h^{2}}{\lambda v}=-\frac{\rho v h^{2}}{\lambda} \frac{\partial P}{\partial x}$

We assume that the fluid with pollutants is isotropic and homogenous so that molecular diffusivity $\mathrm{D}$, viscosity $\mu$ are all constants.

$\frac{d^{4} u}{d y^{4}}-a^{2} \frac{d^{2} u}{d y^{2}}-a^{2} W e P_{e} \vec{E}_{x}=a^{2} P$

where we $=\frac{\varepsilon_{0} V^{2}}{\mu \nu}, P=\frac{-\partial P}{\partial x}, l=\sqrt{\frac{\lambda}{\mu}}, a=\frac{h}{l}$

is the couple stress parameter.

Equation (5) becomes, $\nabla . \vec{J}=0$

using equation (1) we get,

$\sigma\left(\nabla^{2} \phi\right)+\nabla \phi \cdot \nabla \sigma=0$

The boundary conditions on velocity, couple stress and electric potential after dimensionless are

$\mathrm{u}=0$ at $\mathrm{y}=0,1$

$\frac{d^{2} u}{d y^{2}}=0$ at $y=0,1$

$\left.\begin{array}{l}\phi=x \text { at } y=0 \\ \phi=x-x_{0} \text { at } y=h\end{array}\right\}$

The solution for $\phi$, according to (14)depends on $\sigma$ which in turn depends on the temperature $\mathrm{Tb}$ as in (12). In a poorly conducting fluid, $\sigma<<1$ and hence any perturbation on it is negligible and hence it depends on the conduction temperature Tb namely, 
$\frac{d^{2} T_{b}}{d y^{2}}=0$

with the boundary conditions

$\left.\begin{array}{c}T_{b}=T_{0} \text { at } y=0 \\ T_{b}=T_{1} \text { at } y=h\end{array}\right\}$

is $\mathrm{T}_{\mathrm{b}}-\mathrm{T}_{0}=\Delta \mathrm{T} y$

Therefore equation (12) becomes

$\sigma=\sigma_{0}\left[1+\alpha_{\mathrm{h}} \Delta \mathrm{Ty}\right]=\sigma_{0}(1+\alpha \mathrm{y})=\sigma_{0} \mathrm{e}^{\alpha \mathrm{y}}$

$\sigma \approx \mathrm{e}^{\alpha \mathrm{y}}($ since $\alpha<<1)$

where $\alpha=\alpha_{\mathrm{h}} \Delta \mathrm{T}$

Then (14) using (21) we get

$\frac{d^{2} \phi}{d y^{2}}+\alpha \frac{d \phi}{d y}=0$

Its solution satisfying the boundary condition (11) is

$\phi=x-\frac{x_{0}}{1-e^{-\alpha}}\left[1-e^{-\alpha y}\right]$

using the dimensionless quantities and equation (23), equation (6), (7) and (8) reduce to $\rho \mathrm{e}=\nabla \cdot \overrightarrow{\mathrm{E}}=-\nabla^{2} \phi=$ $\frac{x_{0} \alpha^{2} e^{-\alpha y}}{1-e^{-\alpha}}, \mathrm{E}_{\mathrm{x}}=-1$

$\rho_{\mathrm{e}} \mathrm{E}_{\mathrm{x}}=\frac{x_{0}^{2} \alpha^{2} e^{-\alpha y}}{1-e^{-\alpha}}$

\section{ELECTRO HYDRODYNAMIC DISPERSION OF AEROSOLS}

The solution of equation (13) satisfying the condition (9) is

$\mathrm{u}=\mathrm{k}_{1} \mathrm{k}_{2} \mathrm{y}-\mathrm{k}_{3} \mathrm{k}_{2} \mathrm{y}+\mathrm{k}_{4}\left(\mathrm{y}-\mathrm{y}^{2}\right)-\mathrm{k}_{1}+\mathrm{k}_{1} \mathrm{e}^{\alpha \mathrm{y}}+\mathrm{k}_{3}-\mathrm{k}_{3} \mathrm{e}^{\mathrm{ay}}-\mathrm{k}_{5}+\mathrm{k}_{5} \mathrm{e}^{\mathrm{ay}}$

$-\mathrm{k}_{6} 2$ sinhay

The average velocity is given by,

$\bar{u}=\frac{1}{2} \int_{0}^{1} u d y=\frac{k_{1} k_{2}}{4}-\frac{k_{3} k_{2}}{4}+\frac{k_{4}}{4}-\frac{k_{4}}{6}$

$-k_{1} k_{7}+k_{3} k_{8}-k_{5} k_{8}-k_{6} k_{9}$
Now $\mathrm{C}$, the concentration of aerosol in the atmosphere which diffuse in a fully developed flow, can be written as

$\frac{\partial C}{\partial t}+u \frac{\partial C}{\partial x}=D\left(\frac{\partial^{2} C}{\partial x^{2}}+\frac{\partial^{2} C}{\partial y^{2}}\right)-K_{1} C$

where $K_{1}$ is the reaction rate parameter. To find the concentration distribution, we assume following Taylor (1953), the longitudinal diffusion is very much less than the transverse diffusion which implies.

$\frac{\partial^{2} C}{\partial x^{2}}<<\frac{\partial^{2} C}{\partial y^{2}}$

This small transport and the small longitudinal concentration gradient must be proportional to one another. Thus the combined effect of longitudinal advection and transverse diffusion is to disperse the aerosol longitudinally relative to a frame moving at the mean speed of flow by a mechanism which obeys the same law as on ordinary one dimensional diffusion relative to fluid at rest. Then equation (27) takes the form.

$\frac{\partial C}{\partial t}+u \frac{\partial C}{\partial x}=D \frac{\partial^{2} C}{\partial y^{2}}-K_{1} C$

The non-dimensional boundary conditions on concentrations are

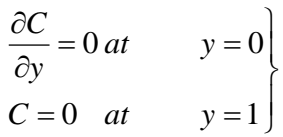

We now introduce the following dimensions variables,

$x^{*}=\frac{x}{h} ; y^{*}=\frac{y}{h} ; C^{*}=\frac{C}{C_{0}} ; t^{*}=\frac{t}{\bar{t}} ; \xi=\frac{\bar{x}-u t}{L} ; \beta^{2}=\frac{h^{2}}{D} K_{1}$

where $\mathrm{L}$ is the characteristic length along the flow direction. Equation (26) in non-dimensionless form can be written as

$\frac{1}{\bar{t}} \frac{\partial C}{\partial t}+\frac{V}{L} \frac{\partial C}{\partial \xi}=\frac{D}{h^{2}} \frac{\partial^{2} C}{\partial y^{2}}-K_{1} C$

$V=u-\bar{u}=\left(k_{1} k_{2}-k_{3} k_{2}+k_{4}\right) y-k_{4} y^{2}+k_{1} e^{-\alpha y}$
$+e^{a y}\left(k_{5}-k_{3}-k_{6}\right)+k_{6} e^{-\alpha y}+k_{10}$ 
Following Taylor (1953) we assume partial equilibrium at any cross section of the layer and obtain $\mathrm{C}$ as the variation of $\mathrm{y}$ by approximating equation (30) in the form

$\frac{\partial^{2} C}{\partial y^{2}}-\beta^{2} C=Q V$

where

$Q=\frac{h^{2}}{D L} \frac{\partial C}{\partial \xi}$

The solution of equation (32) using the equation (31) and satisfying the boundary conditions (29) we get

$$
\begin{aligned}
& c=Q\left\{c_{1} e^{\beta y}+c_{2} e^{-\beta y}-\frac{k_{11}}{\beta^{2}} y+\frac{k_{4}}{\beta^{2}} y^{2}+\frac{1}{\alpha^{2}-\beta^{2}} k_{1} e^{-\alpha y}\right. \\
& \left.+\frac{1}{a^{2}-\beta^{2}} k_{12} e^{a y}++\frac{1}{a^{2}-\beta^{2}} k_{6} e^{-a y}+k_{13}\right\}
\end{aligned}
$$

The volumetric rate at which the fluid is transported across a section of a layer of unit breadth is

$$
M=h \int_{0}^{1} C V d y
$$

using equations (33) and (31) we get,

$$
M=\frac{h^{3}}{D L} G \frac{\partial C}{\partial \xi}
$$

Following Taylor (1953) we assume that the variation of C with $\xi$ are small compared with those in the longitudinal direction and $\mathrm{C}_{\mathrm{m}}$ is the mean concentration over a section, $\frac{\partial C}{\partial \xi} \mathrm{is}$ indistinguishable from $\frac{\partial C_{m}}{\partial \xi}$ so that (35) can be written as

$M=\frac{h^{3}}{D L} G \frac{\partial C_{m}}{\partial \xi}$

The fact that no material is lost in the process is expressed by the continuity equation for $\mathrm{C}_{\mathrm{m}}$ namely

$$
\frac{\partial M}{\partial \xi}=-\frac{2}{L} \frac{\partial C_{m}}{\partial t}
$$

where $\partial / \partial \mathrm{t}$ represents differentiation with respect to time at point where $\xi$ is constant.

Equation (36) using (37)

$$
-\frac{2}{L} \frac{\partial C_{m}}{\partial t}=\frac{h^{3}}{D L} G \frac{\partial^{2} C_{m}}{\partial \xi^{2}}
$$

$\frac{\partial C_{m}}{\partial t}=D * \frac{\partial^{2} C_{m}}{\partial \xi^{2}}$

where

$D^{*}=-\frac{h^{3}}{2 D} G$

which is the equation governing the longitudinal dispersion. Equation (38) implies that $C_{m}$ is dispersed relative to a plane which moves with average velocity $\bar{u}$ exactly asthough, it has been diffused by a process which obeys the same law as the molecular diffusion.

\section{CONCLUSION}

Pollutants emitted from different surface sources are mixed with the air of the earth's atmosphere. Gaseous constituents of the atmosphere are continuously being exchanged with vegetation, the ocean and biological organisms. Cycles of the atmospheric gases involve a number of physical and chemical processes within atmosphere itself, by biological activities. Various removal mechanisms are known to occur in the atmosphere which are effective in removing pollutants. Here the atmospheric gas is modeled as couple stress fluid.

In this paper, the axial dispersion in a couple stress fluid bounded by electrodes in the presence of chemical reaction is studied using Taylor dispersion model. The Taylor dispersion coefficient is determined and numerically computed for different values of electric number and the results are represented graphically.

Figure 2 represents the effect of electric number on the velocity profiles of couple stress fluid and aerosols. It is observed that the velocity increases with increase in electric number.

Figure 3 represents the effect of electric number $W_{e}$ on dispersion of aerosols in couple stress fluid for different reaction rate $\beta$. It shows that the $D^{*}$ increases with an increase in $W_{e}$ but decreases with an increase in $\beta$. Figure 4 shows that the $D^{*}$ decreases with increase in $\beta$ and couple stress parameter $a$. Figure 5 shows that the $D^{*}$ increases with increase in $W_{e}$ and decreases with an increase in $a$. From these figures it is found that the electric field enhances the transport of aerosols and the first order chemical reaction decreases the transport of aerosols. That is it removes efficiently solid or liquid particles from gases discharged to the environment. The proposed model and analysis presented here also suggests that to remove the aerosols from the atmosphere, external species may be introduced in the atmosphere which can interact with the pollutant and remove it by some suitable removal processes based upon the physical and chemical properties of the pollutants as well as that of externally introduced species. 


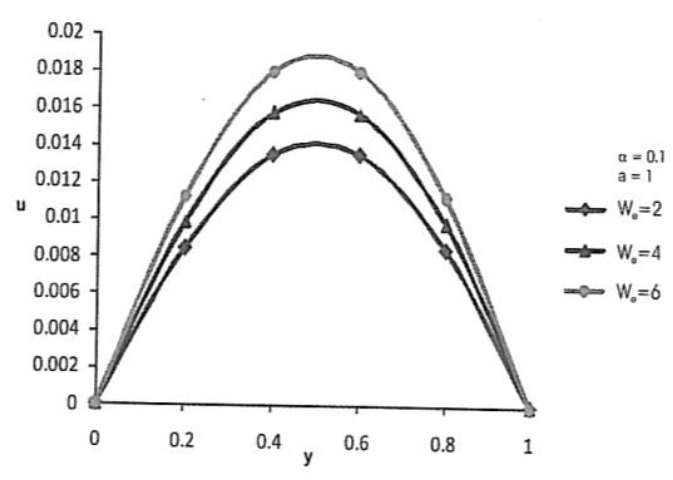

Figure 2. Velocity profiles of aerosols for different electric numbers.

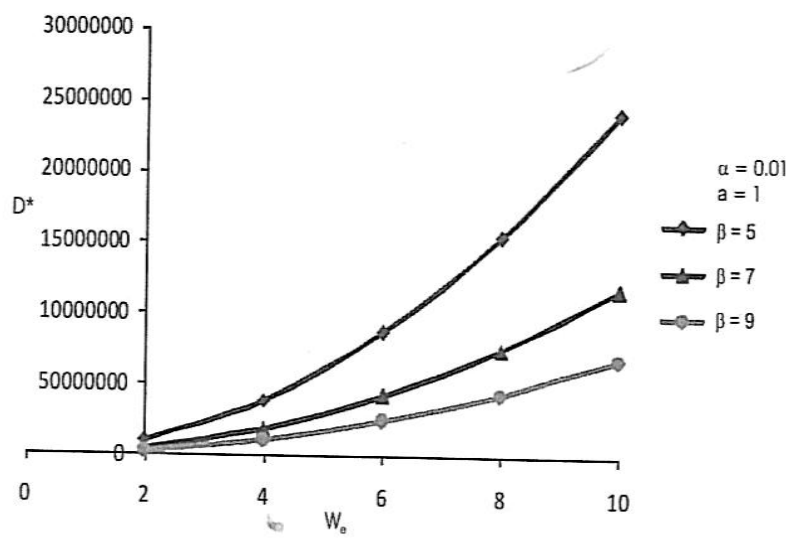

Figure 3. Effect of reaction rate $\beta$ on dispersion coefficient $\left(D^{*}\right)$ with electric number $W_{e}$

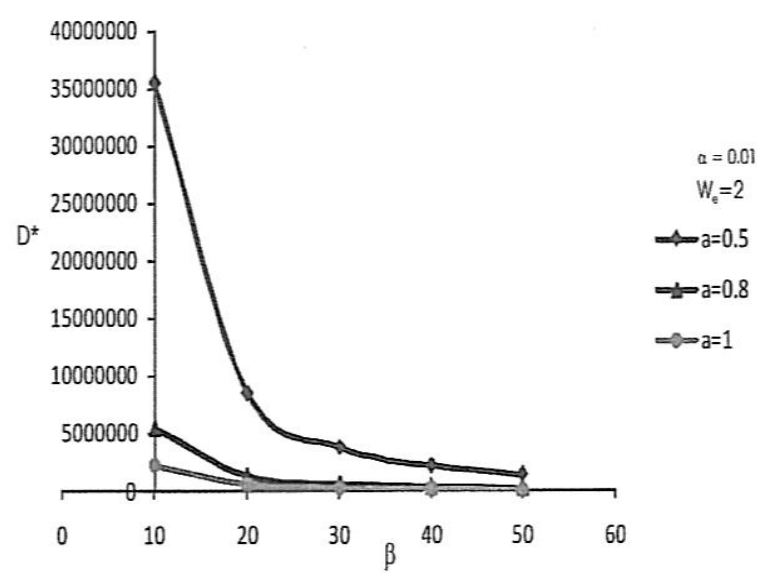

Figure 4. Effect of couple stress $a$ on dispersion coefficient $\left(D^{*}\right)$ witl reaction rate $\beta$

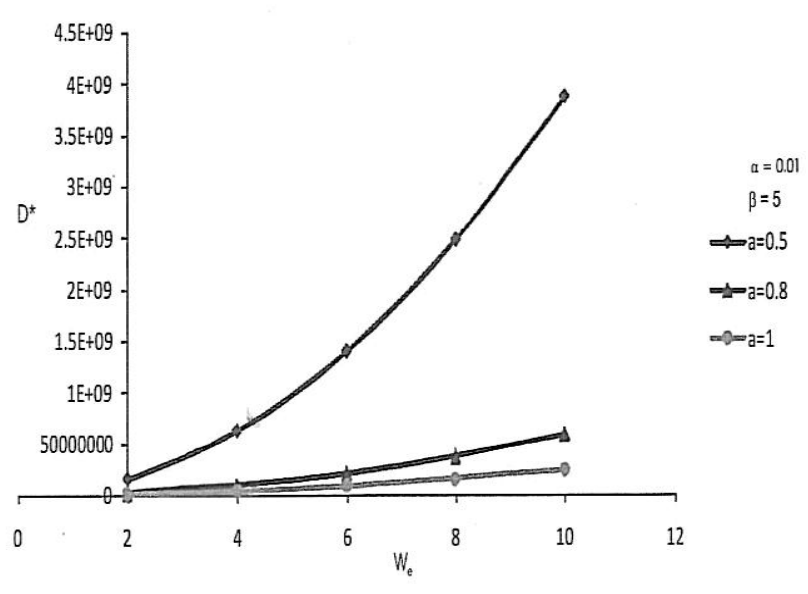

Figure 5. Dispersion coefficient $\left(D^{*}\right)$ versus electric number $\left(W_{e}\right)$ for

$$
\text { different values of } a
$$

\section{REFERENCES}

[1] Brimlecombe, P.(1996). Air pollution and chemistry, Cambridge University Press, Cambridge.

[2] Chiu - on, NG., Rudraiah, N., Nagraj, C., and Nagaraj. H.N. Electrohydrodynamic dispersion of macromolecular components in nanostructured biological bearing. Journal of Energy. Heat and Mass Tranfer, Vol. 27, (2005) pp. 39.

[3] Jayaratne, E.R. and Verma, T.S. Environmental aerosols and their effect on the earth's local fair weather electric field. J. Meteorol, Atmospheric physics, 86, 3(4) (2004), 275-280.

[4] Jacob, D.J. Heterogenous chemistry and trophosphere ozone. Atmospheric environment, 34 (12-14), (2000), 2131.

[5] Kvetoslav. R., Spurny. Aerosol chemical processes in the environment, Qc882, 42(2000) A32.

[6] Malkus, W.V.R and Veronis G. Surface electroconvection. Physics of Fluids. 4(1), (1961)13.

[7] Nirmala P. Ratchagar., Meena Priya P. Effect of couple stress on the unsteady convective diffusion of atmospheric aerosols in the presence of electric field International Journal of Mathematical Sciences and Engineering Applications 5(4), (2011), 293-310.

[8] Rudraiah N., and Ng,C.O. A model for manufacture of nano-sized smart materials free from impurities. Review Articles, current Sci., 86, 8 (2004) 1076.

[9] Stokes, V.K., Couple stress in fluids. Phys. Fluids. 9 (1966) pp.1709-1715.

[10] Taylor G.I. Dispersion of soluble matter in solvent flowing slowly through a tube. Proc. Roy. Soc. London. A. 219 (1953) pp.186-203. 


\section{APPENDIX}

$$
\begin{aligned}
& a_{0}=\left(\frac{W_{e} x_{0} \alpha^{2}}{1-e^{-\alpha}}\right) ; k_{1}=\left(\frac{a_{0}}{\alpha^{2}\left(\alpha^{2}-a^{2}\right)}\right) ; \\
& k_{2}=\left(1-e^{-\alpha}\right) ; k_{3}=\left(\frac{a_{0}}{a^{2}\left(\alpha^{2}-a^{2}\right)}\right) ; k_{4}=\frac{P}{2} ; \\
& k_{5}=\frac{P}{a^{2}} \text {; } \\
& k_{6}=\frac{1}{2(\sin h a)}\left(\frac{a_{0}\left(e^{-\alpha}-e^{a}\right)}{a^{2}\left(\alpha^{2}-a^{2}\right)}+\frac{a^{2} P\left(e^{a}-1\right)}{a^{4}}\right) ; \\
& k_{7}=\left(\frac{\alpha+1+e^{-\alpha}}{2 \alpha}\right) ; k_{8}=\left(\frac{a-1-e^{a}}{2 a}\right) ; \\
& k_{9}=\left(\frac{1+(\cos h a)}{a}\right) \text {; } \\
& k_{10}=\left(-k_{1}+k_{3}-k_{5}-\frac{k_{1} k_{2}}{4}+\frac{k_{3} k_{2}}{4}-\frac{k_{4}}{4}\right. \\
& \left.+\frac{k_{4}}{6}+k_{1} k_{7}-k_{3} k_{8}+k_{5} k_{8}+k_{6} k_{9}\right) \text {; } \\
& k_{11}=\left(k_{1} k_{2}-k_{3} k_{2}+k_{4}\right) ; k_{12}=\left(k_{5}-k_{3}-k_{6}\right) \text {; } \\
& k_{13}=\left(\frac{2 k_{4}}{\beta^{4}}-\frac{k_{10}}{\beta^{2}}\right) \text {; } \\
& c_{1}=\left(\frac { 1 } { 2 \beta \operatorname { c o s h } \beta } \left(\frac{k_{11}\left(e^{-\beta}+\beta\right)}{\beta^{2}}-\frac{k_{4} \beta}{\beta^{2}}\left(1+\frac{2}{\beta^{2}}\right)\right.\right. \\
& -\frac{k_{1}}{\alpha^{2}-\beta^{2}}\left(\beta e^{-\alpha}-\alpha e^{-\beta}\right)-\frac{k_{12}}{a^{2}-\beta^{2}}\left(\beta e^{a}-a e^{-\beta}\right) \\
& \left.-\frac{k_{6}}{a^{2}-\beta^{2}}\left(\beta e^{-a}-a e^{-\beta}\right)-\frac{k_{10}}{\beta}\right) \text {; } \\
& c_{2}=\left(\frac { 1 } { \beta } \left(\frac { 1 } { 2 \operatorname { c o s h } \beta } \left(\frac{k_{11}\left(e^{-\beta}+\beta\right)}{2 \beta \cosh \beta}-\frac{k_{4} \beta}{\beta^{2}}\left(1+\frac{2}{\beta^{2}}\right)\right.\right.\right. \\
& -\frac{k_{1}}{\alpha^{2}-\beta^{2}}\left(\beta e^{-\alpha}-\alpha e^{-\beta}\right)-\frac{k_{12}}{a^{2}-\beta^{2}}\left(\beta e^{a}-a e^{-\beta}\right) \\
& \left.-\frac{k_{6}}{a^{2}-\beta^{2}}\left(\beta e^{-a}-a e^{-\beta}\right)-\frac{k_{10}}{\beta}\right)-\frac{k_{11}}{\beta^{2}}-\frac{\alpha k_{1}}{\alpha^{2}-\beta^{2}} \\
& \left.-\frac{a k_{12}}{a^{2}-\beta^{2}}-\frac{a k_{6}}{a^{2}-\beta^{2}}\right) \text { ); }
\end{aligned}
$$

$$
b_{0}=\left(k_{11} k_{13}-\frac{k_{10} k_{11}}{\beta^{2}}\right)
$$$$
b_{1}=\left(-k_{13} k_{4}-\frac{k_{11}{ }^{2}}{\beta^{2}}+\frac{k_{10} k_{4}}{\beta^{2}}\right)
$$$$
b_{2}=\left(k_{12} k_{13}+\frac{k_{10} k_{12}}{a^{2}-\beta^{2}}\right)
$$$$
b_{3}=\left(-\frac{k_{11} k_{12}}{\beta^{2}}+\frac{k_{11} k_{12}}{a^{2}-\beta^{2}}\right) \text {; }
$$$$
b_{4}=\left(\frac{k_{12} k_{4}}{\beta^{2}}-\frac{k_{12} k_{4}}{a^{2}-\beta^{2}}\right) \text {; }
$$$$
b_{5}=\left(\frac{k_{4} k_{6}}{\beta^{2}}-\frac{k_{4} k_{6}}{a^{2}-\beta^{2}}\right) \text {; }
$$$$
b_{6}=\left(k_{1} k_{13}+\frac{k_{1} k_{10}}{\alpha^{2}-\beta^{2}}\right) \text {; }
$$$$
b_{7}=\left(-\frac{k_{1} k_{11}}{\beta^{2}}+\frac{k_{1} k_{11}}{\alpha^{2}-\beta^{2}}\right) \text {; }
$$$$
b_{8}=\left(\frac{k_{1} k_{12}}{a^{2}-\beta^{2}}+\frac{k_{1} k_{12}}{\alpha^{2}-\beta^{2}}\right)
$$$$
b_{9}=\left(\frac{k_{1} k_{4}}{\beta^{2}}-\frac{k_{1} k_{4}}{\alpha^{2}-\beta^{2}}\right) \text {; }
$$ 


$$
\begin{aligned}
& b_{10}=\left(\frac{k_{1} k_{6}}{a^{2}-\beta^{2}}+\frac{k_{1} k_{6}}{\alpha^{2}-\beta^{2}}\right) ; \\
& G=\left(\frac{b_{0}}{2}+\frac{b_{1}}{3}+\frac{b_{5}\left(2-\left(2+2 a+a^{2}\right) e^{-a}\right)}{a^{3}}+\frac{b_{2}\left(-1+e^{a}\right.}{a}\right. \\
& +\frac{b_{3}\left(1+(-1+a) e^{a}\right)}{a^{2}}+\frac{b_{4}\left(-2+\left(2-2 a+a^{2}\right) e^{a}\right)}{a^{3}} \\
& +k_{10} k_{13}+\frac{\left(1-e^{-a}\right)\left(k_{13} k_{6}\right)}{a}-\frac{b_{8}-b_{8} e^{a-\alpha}}{a-\alpha}+\frac{b_{6}-b_{6} e^{-\alpha}}{\alpha} \\
& +\frac{b_{10}-b_{10} e^{-a-\alpha}}{a+\alpha}+\frac{b_{7}\left(1-e^{-\alpha}(1+\alpha)\right)}{\alpha^{2}} \\
& +\frac{b_{9}\left(2-e^{-\alpha}\left(2+2 \alpha+\alpha^{2}\right)\right)}{\alpha^{3}}-\frac{c_{2}\left(1-e^{a-\beta}\right) k_{12}}{a-\beta} \\
& +\frac{c_{1}\left(1-e^{-a+\beta}\right) k_{6}}{a-\beta}+\frac{c_{1}\left(1-e^{-a+\beta}\right) k_{1}}{\alpha-\beta}+\frac{k_{11} k_{4}}{2 \beta^{2}} \\
& -\frac{k_{4}{ }^{2}}{5 \beta^{2}}-\frac{\left(1-(1+a) e^{-a}\right) k_{11} k_{6}}{a^{2} \beta^{2}}+\frac{\left(c_{1} k_{11}\left(1+e^{\beta}(-1+\beta)\right)\right.}{\beta^{2}}
\end{aligned}
$$$$
+\frac{c_{2}\left(1-e^{-\beta}\right) k_{10}}{\beta}+\frac{c_{1}\left(-1+e^{\beta}\right) k_{10}}{\beta}+\frac{c_{1}\left(-1+e^{a+\beta}\right) k_{12}}{(a+\beta)}
$$$$
+\frac{c_{2}\left(1-e^{-a-\beta}\right) k_{6}}{(a+\beta)}+\frac{c_{2}\left(1-e^{-\alpha-\beta}\right) k_{1}}{(\alpha+\beta)}
$$$$
+\frac{\left(1-(1+a) e^{-a}\right) k_{11} k_{6}}{a^{2}\left(a^{2}-\beta^{2}\right)}+\frac{2 k_{12} k_{6}}{a^{2}-\beta^{2}}+\frac{\left(-1+e^{2 a}\right) k_{12}^{2}}{2\left(a^{3}-a \beta^{2}\right)}
$$$$
+\frac{\left(1-e^{-a}\right) k_{10} k_{6}}{a^{3}-a \beta^{2}}+\frac{c_{2} k_{11}\left(1-e^{-\beta}(1+\beta)\right)}{\beta^{2}}
$$$$
-\frac{c_{1} k_{4}\left(-2+e^{\beta}\left(2-2 \beta+\beta^{2}\right)\right)}{\beta^{3}}
$$$$
-\frac{c_{2} k_{4}\left(2-e^{-\beta}\left(2+2 \beta+\beta^{2}\right)\right)}{\beta^{3}}
$$$$
\left.+\frac{e^{-a_{k} 2} \sinh a}{a^{3}-a \beta^{2}}+\frac{e^{-a_{k} 2} \sinh a}{\alpha^{3}-\alpha \beta^{2}}\right) .
$$ 